\title{
Influência da Espessura do Filme Polimérico Intermediário na Resistência Mecânica de Juntas Híbridas de Alumínio 2024-T3 e CF-PPS Produzidas por União Pontual por Fricção
}

\author{
Natália M. André ${ }^{1}$, Seyed M. Goushegir², Jorge F. dos Santos², Leonardo B. Canto', Sergio T. Amancio-Filho²,3 \\ 1 Universidade Federal de São Carlos - UFSCar, Programa de Pós-graduação em Ciência e Engenharia de Materiais - PPG-CEM, \\ São Carlos, SP, Brasil. \\ 2 Helmholtz-Zentrum Geesthacht, Centre for Materials and Coastal Research, Institute of Materials Research, Materials \\ Mechanics, Solid State Joining Processes, Geesthacht, Germany. \\ 3 Hamburg University of Technology, Institute of Polymer Composites, Hamburg, Germany.
}

Recebido: 03 Nov., 2015

Aceito: 04 Jan., 2016

E-mails: nat.manente@gmail.com (NMA), sergio.amancio@hzg.de (STAF)
Resumo: A União Pontual por Fricção (FSpJ) é uma técnica inovadora para união de estruturas híbridas metal-polímero e baseia-se na geração de calor por fricção. Juntas de alumínio 2024-T3 e compósito laminado de poli(sulfeto de fenileno) com fibra de carbono (CF-PPS) foram produzidas com filme intermediário de PPS. Duas espessuras de filme foram investigadas: 100 e $500 \mu \mathrm{m}$. Ensaios de cisalhamento sob tração demonstraram que as juntas com filmes de $100 \mu \mathrm{m}$ são mais resistentes $(2093 \pm 180 \mathrm{~N})$ em relação às juntas com filme de $500 \mu \mathrm{m}$ (708 \pm 69 N). Adicionalmente, as superfícies de fratura das juntas revelaram áreas de união maiores para as juntas com filmes de $100 \mu \mathrm{m}$ (53 \pm 2 contra $40 \pm 1 \mathrm{~mm}^{2}$ ). Para o filme mais fino, a extensão do amolecimento devido ao calor friccional é maior. Consequentemente, a baixa viscosidade atingida com a fusão do polímero favorece a molhabilidade das superfícies dos componentes da junta pelo PPS amolecido, resultando em melhor adesão entre as partes. Ademais, análises microestruturais demonstraram que a formação do cerne metálico e a interdifusão das moléculas de PPS entre compósito e filme também são favorecidas. Portanto, concluiu-se que a adição do filme mais fino produziu juntas mais resistentes.

Palavras-chave: União pontual por fricção; Estruturas híbridas; Filme intermediário; Resistência mecânica.

\section{Influence of the Interlayer Film Thickness on the Mechanical Performance of AA2024-T3/CF-PPS Hybrid Joints Produced by Friction Spot Joining}

\begin{abstract}
Friction Spot Joining (FSpJ) is an innovative friction-based joining technique for metalpolymer hybrid structures. Friction spot joints of aluminum alloy 2024-T3 and carbon-fiber reinforced poly(phenylene sulfide) composite laminate (CF-PPS) were produced with an additional PPS film interlayer. Two different film thicknesses were investigated in this study: 100 and $500 \mu \mathrm{m}$. Lap shear testing demonstrated that the joints produced with $100 \mu \mathrm{m}$ film $(2093 \pm 180 \mathrm{~N})$ were stronger than the joints with $500 \mu \mathrm{m}(708 \pm 69 \mathrm{~N})$. Additionally, the fracture surface analysis revealed a larger bonding area for the joints with $100 \mu \mathrm{m}$ film $\left(53 \pm 2 \mathrm{~mm}^{2}\right)$ as compared to the joints with $500 \mu \mathrm{m}$ film $\left(40 \pm 1 \mathrm{~mm}^{2}\right)$. Considering the low thermal conductivity of PPS, the thinnest film is more likely to soften by the frictional heat during the joining process. Hence, the low viscosity of the molten PPS favors the wettability of the parts' surface. Microstructural analyses proved that the metallic nub formation and the interdiffusion of PPS chains between film and composite matrix are also favored for thinner film use. Thus, superior adhesion between the partners is achieved. Therefore, it was concluded that the addition of the thinnest film interlayer leads to stronger joints.
\end{abstract}

Key-words: Friction spot joining; Hybrid structures; Film interlayer; Mechanical strength.

\section{Introdução}

Nas últimas décadas, a seleção e o desenvolvimento de novos materiais tornaram-se fatores essenciais para o sucesso e competitividade de importantes setores industriais, tais como o aeronáutico e o automotivo [1]. Engenheiros e designers são cada vez mais confrontados com novas e rigorosas políticas econômicas e ambientais. A redução do
Este é um artigo publicado em acesso aberto (Open Access)
sob a licença Creative Commons Attribution Non-Commerci que permite uso, distribuição e reprodução em qualquer meio, sem restriçōes desde que sem fins comerciais e que 0 trabalho original seja corretamente citado. 
Influência da Espessura do Filme Polimérico Intermediário na Resistência Mecânica de Juntas Híbridas de Alumínio 2024-T3 e CF-PPS Produzidas por União Pontual por Fricção

consumo de combustíveis e da emissão de $\mathrm{CO}_{2}$ estão entre as exigências mais comuns. Uma solução promissora no intuito de suprir tais exigências encontra-se no conceito de estruturas leves híbridas, que combina ligas metálicas leves e compósitos poliméricos. Tal fato justifica-se principalmente pela leveza e excelente resistência mecânica específica associada a esses materiais [2].

Recentemente, o desenvolvimento de estruturas leves híbridas tornou-se o foco das indústrias no setor de transporte. Na indústria aeronáutica, por exemplo, o Boeing 787 tem sua estrutura composta por mais de $50 \%$ em massa de compósitos poliméricos avançados; uma diminuição de $20 \%$ do peso total da aeronave em comparação à estrutura convencionalmente construída em alumínio [3]. Nos últimos 30 anos, a Airbus também tem investigado o uso de polímeros reforçados com fibra de carbono em suas aeronaves. Aplicações tem sido desenvolvidas para componentes como asas móveis e cauda vertical do A310, a cauda horizontal do A320, a quilha dos A340-600, a caixa central da asa do A380, além de partes das asas do Falcon 10, ATR 72 e A400M [4]. Ademais, o recente lançamento do A350 XWB criou um novo padrão nesta classe de aeronaves da Airbus. Tal modelo apresentou uma redução no consumo de combustível de $25 \%$ devido ao uso de $53 \%$ de compósitos poliméricos em sua estrutura [4]. No cenário brasileiro, a Embraer também tem investido em polímeros reforçados com fibra de carbono. $O$ recente lançamento da aeronave militar de transporte de carga - o KC-390 - demonstrou pela primeira vez em âmbito nacional o uso de compósitos poliméricos em soluções leves de proteção balística [5]. Adicionalmente, na indústria automotiva, uma nova geração de carros elétricos e híbridos tem sido desenvolvida pela BMW (ex. i3 e i8). Através da substituição do aço, tradicionalmente utilizado, por polímeros reforçados com fibra de carbono e alumínio, os engenheiros da BMW foram capazes de diminuir drasticamente o peso dos automóveis. Além da redução de peso do automóvel propriamente dito, a substituição da estrutura permite uma economia de $50 \%$ em energia e $70 \%$ em água durante seu processo de fabricação [6].

Técnicas de união de materiais dissimilares são requisitadas em diversos estágios do processo de produção dessas estruturas híbridas. $\mathrm{O}$ aprimoramento das técnicas de união disponíveis, bem como o desenvolvimento de novas técnicas de união, são motivos de intensa pesquisa [7-13].

Atualmente, os principais métodos de união para estruturas híbridas são fixação mecânica e colagem por adesivos [2,14]. No entanto, esses métodos tradicionais apresentam algumas limitações. No caso da fixação mecânica tem-se desvantagens relacionadas aos furos passantes dos parafusos ou rebites que funcionam como concentradores de tensão na estrutura, o que pode levar à falha mecânica prematura da junta, além do rebite aumentar o peso estrutural [14]. Considerando o uso de adesivos, são requeridos a cura da resina e extensivo tratamento superficial, tornando o processo caro e longo [15].

A Weld-bonding é uma tecnologia híbrida de união que propõe a combinação de uma técnica de união e a colagem por adesivos. Esta tecnologia geralmente produz juntas com resistência mecânica e rigidez superiores, uma vez que existem dois tipos de mecanismos de união presentes (ancoramento mecânico e forças adesivas). Adicionalmente, melhor uniformidade da distribuição de carga nas juntas é atingida, devido ao aumento da área de união proporcionado pelo uso do adesivo. Assim, aumenta-se a ductilidade e a vida útil da junta em fadiga, além de geralmente melhorar sua resistência à corrosão [16]. Xu et al. [17] investigaram o efeito da tecnologia weld-bonding em juntas dissimilares de AZ31 e aço HSLA unidas através de soldagem pontual por resistência. Com a tecnologia weld-bonding, o alongamento na ruptura das juntas apresentou-se nove vezes superior, enquanto que a vida em fadiga triplicou em relação às juntas unidas unicamente pela soldagem pontual por resistência.

Analogamente à tecnologia weld-bonding, Mitschang et al. [13] investigaram a adição de filmes intermediários poliméricos a juntas híbridas metal-compósito unidas por indução. Durante o processo de união o filme termoplástico é amolecido e reconsolidado, fornecendo forças adesivas à junta. Foram produzidas juntas de $\mathrm{AlMg}_{3} / \mathrm{CF}-\mathrm{PA} 66 \mathrm{e}$ aço DC01/CF-PEEK usando filmes de PA66 e PEEK, respectivamente. A adição do filme intermediário produziu incrementos na resistência ao cisalhamento de $15 \%$ para as juntas de $\mathrm{AlMg}_{3} / \mathrm{CF}-\mathrm{PA} 66$ e $55 \%$ para as juntas de DC01/CF-PEEK.

A União Pontual por Fricção (do Inglês 'Friction Spot Joining - FSpJ') desponta como uma técnica alternativa e inovadora de união para estruturas híbridas. Desenvolvida e patenteada pelo instituto de pesquisas Helmholtz-Zentrum Geesthacht da Alemanha [18], esta nova técnica de união é baseada na geração de calor por fricção e tem como principais vantagens a não utilização obrigatória de materiais de adição e tratamentos superficiais prévios extensivos, além de apresentar ciclos curtos de união [9,19]. 
A produção de juntas de alumínio 2024 e compósito laminado de poli(sulfeto de fenileno) reforçado com tecido de fibra de carbono (CF-PPS) por União Pontual por Fricção foi demonstrada com sucesso por Goushegir et al. [9]. Diferentes tratamentos superficiais foram testados e níveis de até $43 \mathrm{MPa}$ de resistência ao cisalhamento foram alcançados usando jateamento de areia no componente metálico. O processo FSpJ tem sido explorado para união de diversas combinações de materiais. Esteves et al. [20] demonstrou a otimização do processo para juntas de alumínio 6181-T4/CF-PPS. Amancio-Filho et al. [19] apresentou a viabilidade de aplicação do processo FSpJ para unir a liga de magnésio AZ31 com GF-PPS e CF-PPS. Nestes estudos, as juntas híbridas unidas por FSpJ atingiram excelentes níveis de resistência mecânica, com valores superiores ou comparáveis a outras técnicas de união de estruturas híbridas já consolidadas, como a soldagem por resistência e por indução. No entanto, apesar da excelente resistência mecânica atingida, as juntas unidas por FSpJ possuem baixa ductilidade, apresentando alongamentos limitados e falhas catastróficas quando solicitadas estaticamente.

Baseando-se no potencial da tecnologia weld-bonding em aprimorar a ductilidade de suas juntas, a liga de alumínio 2024-T3 e compósito laminado de poli(sulfeto de fenileno) reforçado com tecido de fibra de carbono (CF-PPS) foram unidos por FSpJ com filme intermediário de PPS. Utilizando-se de uma estratégia similar daquela do estudo realizado por Mitschang et al. [13], André et al. [21] demonstraram a viabilidade técnica de se produzir juntas de AA2024-T3/CF-PPS com filme intermediário de PPS através da União Pontual por Fricção. Durante o processo de união o filme intermediário é fundido e recristalizado, intensificando as forças adesivas na junta. Com a adição do filme, a área de união das juntas híbridas é expandida e, consequentemente, são atingidas resistência mecânica, rigidez, alongamento na ruptura e vida em fadiga superiores [21]. Adicionalmente, a utilização do filme termoplástico torna o processo mais rápido e, portanto, vantajoso, já que elimina a longa etapa de cura inerente ao uso dos adesivos termofixos.

No presente estudo, a influência da espessura do filme de PPS intermediário sobre o desempenho mecânico das juntas híbridas AA2024-T3/CF-PPS produzidas por FSpJ foi investigada. Para tanto, foram analisados os mecanismos de união bem como a resistência ao cisalhamento sob tração de juntas híbridas com duas espessuras de filme intermediário: 100 e $500 \mu \mathrm{m}$.

\section{União Pontual por Fricção ('Friction Spot Joining - FSpJ')}

A FSpJ é uma técnica inovadora para produção de juntas híbridas metal-compósito polimérico, desenvolvida e patenteada pelo instituto de pesquisas alemão Helmholtz-Zentrum Geesthacht (HZG) [18]. A FSpJ busca mitigar as desvantagens dos processos de união para estruturas híbridas, os quais mostram-se limitados diante da susceptibilidade à degradação térmica dos polímeros, à danos ao reforço fibroso no caso dos compósitos poliméricos, e à alta dissimilaridade de propriedades dos materiais envolvidos na junta [19-21].

A União Pontual por Fricção utiliza uma ferramenta não-consumível composta por três partes concêntricas - um anel de fixação, uma camisa e um pino - que rotacionam e se movem axialmente e de forma independente (Figura 1). O processo apresenta duas variantes: camisa-penetrante e pino-penetrante [19].

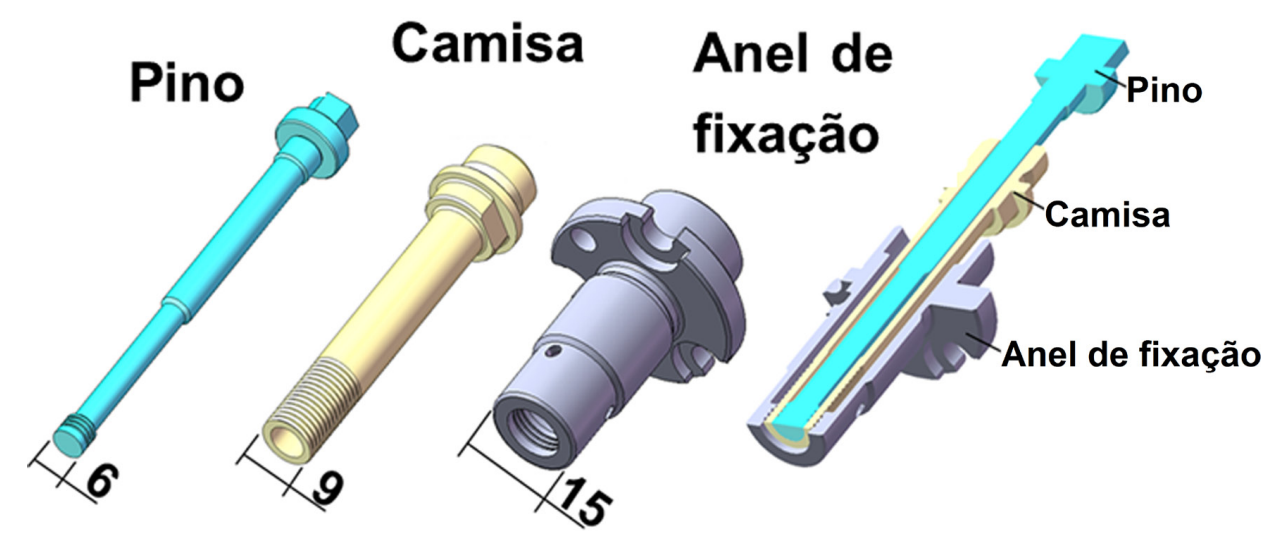

Figura 1. Ferramentas utilizadas no processo FSpJ (dimensões em mm). (Adaptado de Amancio-Filho et al. [19]). 
Para a produção de juntas híbridas metal-polímero, inicialmente os componentes a serem unidos são sobrepostos com auxílio de um porta-amostras [19] e firmemente mantidos pelo anel de fixação e um pistão, de modo que o componente metálico esteja em contato direto com a ferramenta.

De maneira simplificada pode-se dividir o processo FSpJ na variante camisa-penetrante em três etapas (Figura 2) [19]. Na primeira etapa, tem-se a penetração da camisa, que aquece, amolece e deforma o componente metálico por fricção, enquanto o pino é retraído, formando uma cavidade que é preenchida por metal amolecido (Figura 2-1). A penetração da ferramenta se dá somente no material metálico, evitando a deterioração do componente polimérico e danos ao reforço fibroso, no caso do uso de compósitos, que poderiam comprometer o desempenho mecânico da junta [19]. Ao final do tempo de penetração pré-determinado, camisa e pino retornam à posição inicial, ainda rotacionando. Neste movimento, o metal amolecido que se encontrava na cavidade formada pela retração do pino é forçado de volta, preenchendo a inscrição formada pela penetração da camisa (Figura 2-2). No final do processo, a ferramenta é retraída e a junta é consolidada sob pressão (Figura 2-3).

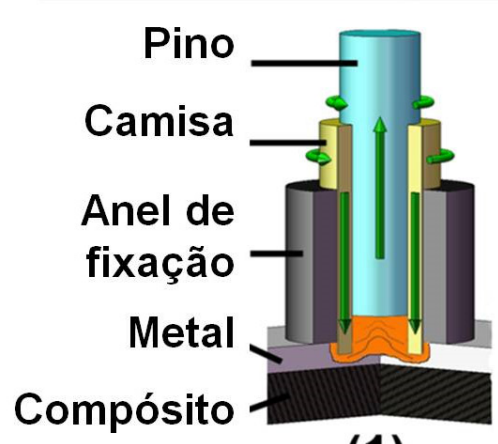

(1)

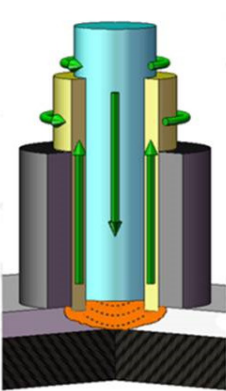

(2)

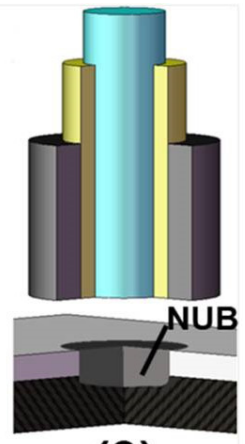

(3)

Figura 2. Esquema simplificado das etapas da FSpJ na variante camisa penetrante. (1) Penetração da camisa, amolecimento e deformação do metal; (2) Retração da camisa e preenchimento do ponto de união; (3) Consolidação da junta. (Adaptado de Amancio-Filho et al. [19]).

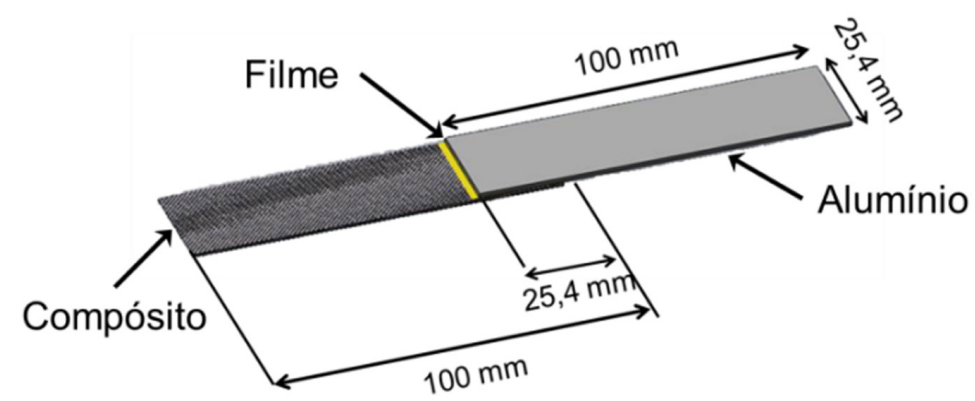

Figura 3. Configuração e dimensões das juntas produzidas por FSpJ.

Na variante pino-penetrante tem-se a penetração do pino no componente metálico, enquanto a camisa é retraída. As outras etapas do processo de união são análogas à variante camisa-penetrante. A principal diferença entre as duas variantes refere-se ao tamanho da área de união, que se apresenta maior na variante camisa-penetrante produzindo juntas mais resistentes [19].

Existem dois mecanismos de união primários responsáveis pela resistência mecânica de juntas híbridas unidas por FSpJ: ancoramento mecânico e forças adesivas [19]. O metal amolecido na primeira etapa do processo é levemente deformado pelo movimento de penetração da ferramenta e essa característica geométrica, chamada "cerne metálico" (do Inglês 'metallic nub'), é responsável pelo macro-ancoramento mecânico entre os componentes da junta. Adicionalmente, parte do calor gerado pela fricção entre a ferramenta e o metal é conduzido através do 
componente metálico para o componente polimérico criando uma fina camada de polímero fundido/amolecido na interface metal-polímero. $O$ posterior resfriamento dessa camada na consolidação da junta fornecerá forças adesivas que também contribuirão para o bom desempenho mecânico das juntas [19].

Os principais parâmetros do processo de união por FSpJ são velocidade de rotação da ferramenta, profundidade de penetração, tempo de penetração e retração da ferramenta (tempo de união) e força de união. Outros parâmetros que podem influenciar a formação da junta são a pressão de fixação do porta-amostras, a geometria e o acabamento superficial das partes a serem unidas, além das propriedades físico-químicas e térmicas dos materiais a serem unidos.

Esteves et al. [22] estudaram a viabilidade técnica da nova tecnologia FSpJ na junção de compósito laminado de poli(sulfeto de fenileno) reforçado com tecido de fibra de carbono (CF-PPS) e liga de alumínio 6181-T4. Excelentes níveis de resistência mecânica foram observados, além de forte influência de tratamentos superficiais pré-união do componente metálico sobre o desempenho mecânico das juntas. A superfície do componente metálico foi levemente lixada (SiC, P1200) para remoção de óxidos naturalmente produzidos pela liga de alumínio. Adicionalmente, em alguns casos, a superfície metálica foi atacada quimicamente com ácido nítrico durante 30 minutos a fim de aumentar a molhabilidade da superfície metálica pelo polímero amolecido. As juntas apresentaram resistência ao cisalhamento de aproximadamente $11 \mathrm{MPa}$ quando apenas lixadas, enquanto que quando submetidas ao ataque químico prévio as juntas atingiram resistência significativamente maior, cerca de $29 \mathrm{MPa}$.

Esteves et al. [20] explorou a influência dos parâmetros de processo na microestrutura e resistência mecânica de juntas sobrepostas duplas de Al 6181-T4/CF-PPS unidas por FSpJ. O método Taguchi e a análise de variância (ANOVA) foram empregados e demonstraram que a velocidade de rotação da ferramenta é o parâmetro processual de maior influência sobre a resistência mecânica das juntas, seguida pelo tempo de união.

Goushegir et al. [9] analisaram aspectos microestruturais e desempenho mecânico de juntas de Al 2024-T3/CF-PPS. Os autores demonstraram uma importante correlação entre os parâmetros do processo FSpJ, a área de união entre os componentes e a resistência ao cisalhamento das juntas. Diferentes tratamentos superficiais foram testados e níveis de resistência ao cisalhamento de até $43 \mathrm{MPa}$ foram alcançados usando jateamento de areia no componente metálico.

Apesar dos excelentes níveis de resistência mecânica alcançados nos estudos anteriores, as juntas unidas por FSpJ apresentam baixa ductilidade, resultando em alongamentos limitados e falhas catastróficas quando solicitadas estaticamente. Assim, baseando-se no potencial da tecnologia weld-bonding em aprimorar a ductilidade das juntas, um filme intermediário de PPS foi adicionado às juntas híbridas de AA2024-T3/CF-PPS produzidas por FSpJ. Durante o processo de união o filme intermediário é fundido e recristalizado, intensificando as forças adesivas na junta. Dessa forma, a área de união das juntas é expandida e, consequentemente, são antecipadas maiores resistência mecânica, rigidez, alongamento na ruptura e vida em fadiga superiores.

\section{Materiais e Métodos}

\subsection{Materiais}

A liga de alumínio 2024-T3 foi empregada como componente metálico na produção das juntas deste estudo. Utilizou-se chapas laminadas de $2 \mathrm{~mm}$ de espessura, fornecidas pela empresa Costellium da França. Esta é uma liga endurecível por precipitação e revenida na condição T3 (solubilização, trabalho a frio e envelhecimento natural) largamente utilizada na indústria aeronáutica. A liga apresenta resistência à tração de $435 \mathrm{MPa}$ e temperatura de fusão em torno de $638^{\circ} \mathrm{C}$ [23].

Como componente compósito, foi utilizado um laminado de poli(sulfeto de fenileno) (PPS) reforçado com tecido de fibra de carbono com espessura nominal de $2,17 \mathrm{~mm}$, comercializado como CETEX $^{\circledR}$ pela empresa Tencate da Holanda. O compósito constitui-se de $43 \%$ em peso de fibra de carbono tecidas na configuração $5 \mathrm{H}$ Satin e sobrepostas em sete camadas $[(0 / 90),( \pm 45)]_{3^{\prime}}(0 / 90)$. O CF-PPS apresenta resistência à tração de $790 \mathrm{MPa}$ (Warp)/750 MPa (Weft) e resistência ao cisalhamento interlaminar de $131 \mathrm{MPa}$. As temperaturas de transição vítrea e de fusão do PPS no compósito são $120^{\circ} \mathrm{C}$ e $280^{\circ} \mathrm{C}$, respectivamente. Além das excelentes propriedades mecânicas, este material oferece ótima resistência química e à solventes [24]. 
Influência da Espessura do Filme Polimérico Intermediário na Resistência Mecânica de Juntas Híbridas de Alumínio 2024-T3 e CF-PPS Produzidas por União Pontual por Fricção

Filmes de PPS de 100 e $500 \mu \mathrm{m}$ de espessura fornecidos pela empresa LITE P da Áustria foram utilizados como camada intermediária entre alumínio e compósito. As temperaturas de transição vítrea e de fusão do PPS no filme são $92{ }^{\circ} \mathrm{C}$ e $280^{\circ} \mathrm{C}$, respectivamente [25].

\subsection{Metodologia}

\subsubsection{Produção das juntas}

As juntas foram produzidas em um equipamento de FSpJ (RPS 100, Harms\&Wende) controlado por deslocamento, alocado no centro de pesquisa alemão Helmholtz-Zentrum Geesthacht (HZG). Investigações anteriores $[9,20]$ demonstraram a influência de pré-tratamentos superficiais sobre a resistência mecânica das juntas híbridas. Assim, neste estudo os componentes a serem unidos foram pré-tratados com jateamento de areia $\left(\mathrm{Al}_{2} \mathrm{O}_{3}, 100-150 \mu \mathrm{m}\right)$ por $10 \mathrm{~s}$. A distância entre a pistola e as superfícies jateadas foi de $20 \mathrm{~cm}$. No caso do alumínio, o ângulo de incidência foi $45^{\circ}$ e a pressão de jateamento foi $0,6 \mathrm{MPa}$. Para o compósito, utilizou-se $90^{\circ}$ de ângulo de incidência e 0,3 MPa de pressão no intuito de evitar danos extensivos ao reforço fibroso e aumentar a rugosidade superficial do material. Depois de tratados, os componentes foram limpos mergulhando-os em banho ultrassônico de acetona por 3 minutos e secos com ar comprimido.

Juntas de AA2024-T3/PPS/CF-PPS foram produzidas utilizando combinações de parâmetros com alto e baixo aporte térmico. No entanto, as juntas apresentaram comportamentos similares sob as duas combinações de parâmetros. Por isso, a fim de avaliar a influência da espessura do filme intermediário no desempenho mecânico das juntas a condição de menor aporte térmico foi selecionada. Tal condição de união produz juntas com resistências mecânicas superiores e menores quantidades de defeitos microestruturais. A influência dos parâmetros de FSpJ na resistência mecânica das juntas com filme foi brevemente discutida em André et al. [21]. Neste estudo, utilizou-se a seguinte condição de união: velocidade de rotação da ferramenta de $1900 \mathrm{rpm}$, penetração da camisa de 0,8 mm, tempo de união de 4 s e pressão de união de 0,3 MPa.

As juntas foram produzidas na configuração sobreposta simples ('single-lap') nas dimensões representadas na Figura 3. Três réplicas foram produzidas.

\subsubsection{Monitoramento da temperatura}

Uma termo-câmera (High-end Camera Series ImageIR, Infratech GmbH, Alemanha) de radiação infravermelho foi utilizada para monitorar a temperatura da superfície do alumínio durante o processo de união por FSpJ. Os limites de deteç̧ão da câmera foram configurados na faixa de $150-700^{\circ} \mathrm{C}$, com frequência de $20 \mathrm{~Hz}$.

\subsubsection{Análise microscópica}

As juntas foram cortadas transversalmente, adequadamente embutidas e polidas. As seções transversais foram observadas em microscópio ótico (DM IR microscope, Leica) para investigar os mecanismos de união na interface das juntas.

\subsubsection{Ensaio mecânico}

O desempenho mecânico das juntas foi avaliado através de ensaio de resistência ao cisalhamento sob carregamento de tração. Os ensaios foram realizados em uma máquina universal de ensaios Zwick/Roell 1478 baseado na norma ASTM D3163-01, com velocidade de 1,27 $\mathrm{mm} \mathrm{min}^{-1}$, à temperatura ambiente.

\section{Resultados e Discussões}

\subsection{Evolução da temperatura processual}

A Figura 4 apresenta exemplos da evolução da temperatura na superfície do alumínio durante o processo de união FSpJ para réplicas da condição de união selecionada neste estudo com filme intermediário de $100 \mu \mathrm{m}$ e $500 \mu \mathrm{m}$ de espessura.

O calor friccional é gerado no componente metálico durante o processo FSpJ. Por sua vez, a temperatura processual é medida na superfície do alumínio. Assim, as máximas temperaturas processuais atingidas para as juntas com filmes de $100 \mu \mathrm{m}$ e $500 \mu \mathrm{m}$ para uma mesma condição de união quase não se alteram. A máxima temperatura 


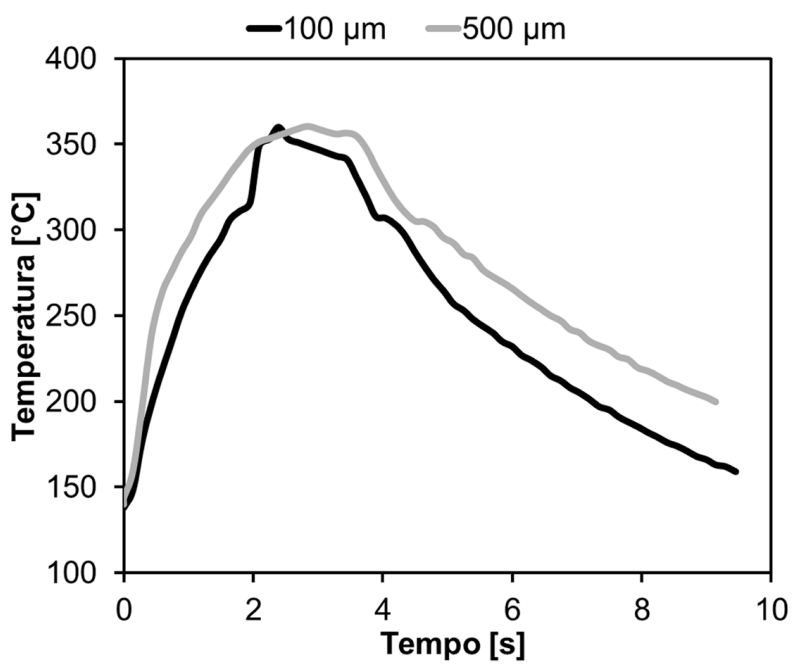

Figura 4. Evolução da temperatura processual na superfície do alumínio durante o processo de união (1900 rpm; $0,8 \mathrm{~mm} ; 4 \mathrm{~s} ; 0,3 \mathrm{MPa})$.

identificada na superfície do componente metálico durante o processo de união foi $360^{\circ} \mathrm{C}$, tanto para as juntas com filmes de $100 \mu \mathrm{m}$ quanto para as juntas com filme de $500 \mu \mathrm{m}$ de espessura (Figura 4). Tal temperatura representa $56 \%$ da temperatura de fusão da liga de alumínio 2024-T3 (638 $\left.{ }^{\circ} \mathrm{C}\right)$. Como discutido por Amancio-Filho et al. [26], devido as altas taxas de cisalhamento (rotação da ferramenta) e temperatura aplicados ao material durante o processo FSpJ, o alumínio na área de união sofre recristalização dinâmica.

O pico de temperatura processual atingida durante o $\mathrm{FSpJ}\left(360^{\circ} \mathrm{C}\right)$ se apresenta acima da temperatura de fusão do PPS da matriz do compósito e do filme intermediário utilizado na produção das juntas $\left(\mathrm{T}_{\mathrm{m}}=280{ }^{\circ} \mathrm{C}\right)$. Consequentemente, o filme de PPS e uma fina camada da matriz polimérica do compósito são amolecidos durante o processo de união, fornecendo forças adesivas às juntas quando reconsolidados. Além disso, é importante notar que a máxima temperatura processual atingida $\left(360^{\circ} \mathrm{C}\right)$ está abaixo da temperatura de início de decomposição térmica do PPS (aproximadamente $450^{\circ} \mathrm{C}$ ) [27-29]. Estudos recentes indicam a possibilidade de recristalização e formação de ligações cruzadas na matriz de PPS e no filme intermediário induzidas pelo processo FSpJ [30]. No entanto, descarta-se a possibilidade de extensiva degradação térmica dos componentes poliméricos das juntas durante a união, visto que o material fundido fica exposto a temperaturas acima de $T_{m}$ por períodos muito curtos (3-4 s).

\subsection{Investigação microestrutural}

A Figura 5 apresenta as seções transversais de juntas unidas por FSpJ com duas espessuras de filmes: (A) $100 \mu \mathrm{m}$ e (B) $500 \mu \mathrm{m}$. A inserção do alumínio no compósito, chamada cerne metálico, é indicado pelas elipses na Figura 5 . O cerne metálico é responsável pelo macro-ancoramento mecânico entre as partes, contribuindo para a resistência mecânica da junta, como reportado por Goushegir et al. [9], Amancio-Filho et al. [19] e Esteves et al. [20].

Observa-se que no caso da junta produzida com filme de espessura mais fina (100 $\mu \mathrm{m})$ o volume metálico deformado durante o processo de união (cerne metálico) se insere levemente no componente compósito estabelecendo efetivo macro-ancoramento mecânico entre metal e compósito (Figura 5A). Por outro lado, no caso da junta produzida com filme de $500 \mu \mathrm{m}$, a formação do cerne metálico se dá pela inserção do metal deformado no filme intermediário, sem atingir o compósito (Figura 5B). Dessa forma, não há efetivo ancoramento mecânico entre o alumínio e o CF-PPS nesta junta.

A Figura 6 mostra detalhes da interface da junta produzida com filme intermediário de $100 \mu \mathrm{m}$ de espessura. Identificou-se a existência de uma linha de separação entre o compósito e o filme intermediário nas extremidades da junta, como mostrado na região I da Figura 5A. Tal linha não é percebida na medida em que se avança da extremidade para o centro da junta, onde maiores temperaturas são atingidas durante o FSpJ. A seta branca na 
(A)

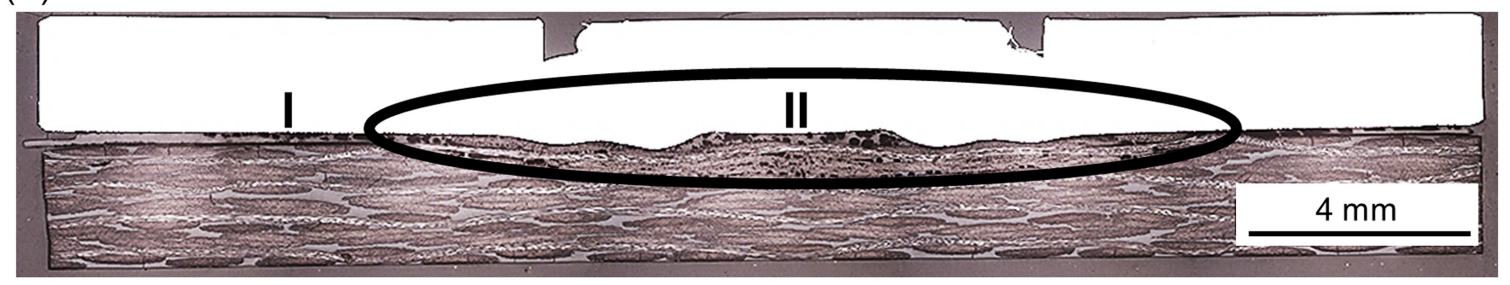

(B)

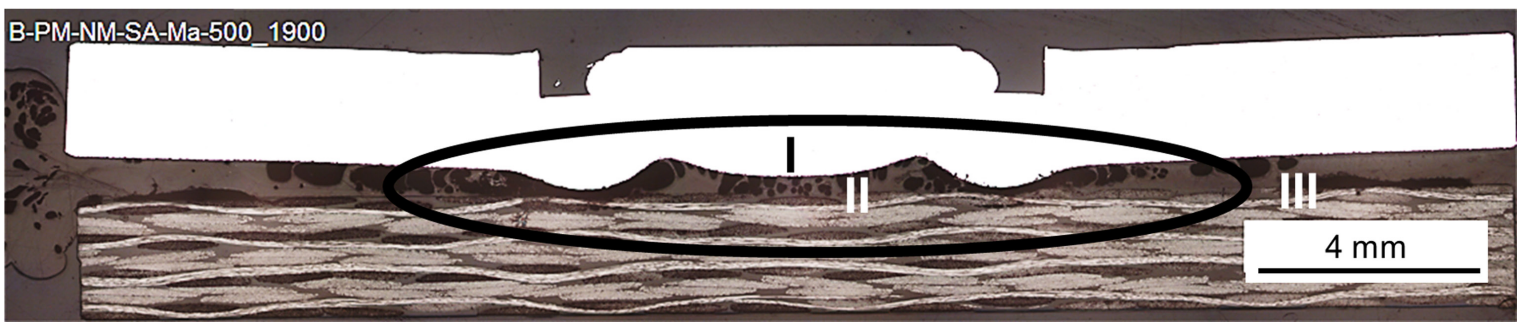

Figura 5. Seção transversal de juntas de AA2024-T3 e CF-PPS com filme intermediário de PPS com espessuras de (A) $100 \mu \mathrm{m}$ e (B) $500 \mu \mathrm{m}$.

(A)

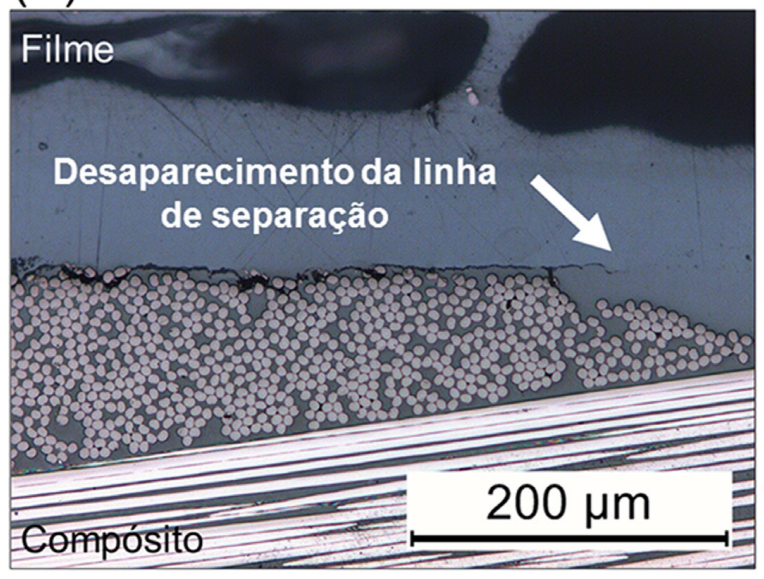

(B)

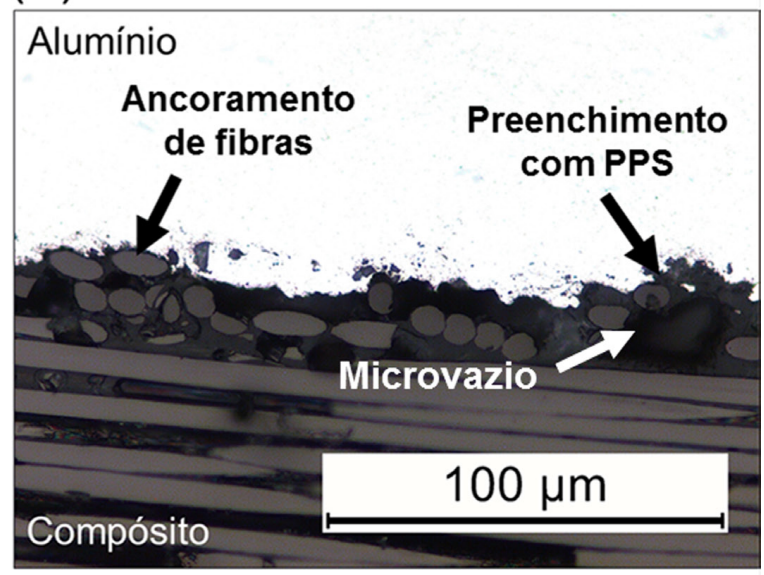

Figura 6. Detalhes microestruturais da interface de juntas unidas por FSpJ com filme intermediário de $100 \mu \mathrm{m}$ de espessura. (A) Região I e (B) região II da Figura 5A.

Figura 6A aponta o fim da linha de separação entre o compósito e o filme. O desaparecimento de tal linha de separação em regiões próximas do centro da junta sugere a interdifusão de cadeias de PPS da matriz do compósito e do filme intermediário, impulsionada pelas altas temperaturas atingidas na região. Dessa forma, garante-se eficiente coesão entre compósito e filme na interface da junta. Como discutido por André et al. [21], durante o processo FSpJ o filme intermediário é aquecido pelo calor friccional, se torna menos viscoso e é expulso do centro para as extremidades da junta pela força axial exercida pela ferramenta, deixando o componente metálico e o compósito em contato direto. No centro da junta (região II da Figura 5A) não é possível identificar a presença do filme intermediário. A Figura 6B mostra o aspecto rugoso adquirido pelo alumínio com o tratamento superficial aplicado. Além disso, observa-se que as irregularidades na superfície metálica são preenchidas com o PPS. Ao mesmo tempo, parte das fibras de carbono expostas pelo tratamento superficial aplicado na superfície do compósito é ancorada na superfície metálica amolecida pelo calor friccional. Ambos micro-mecanismos de ancoramento são importantes contribuintes para a resistência mecânica das juntas, como reportado por Goushegir et al. [9]. 
A Figura 7 apresenta detalhes da interface de juntas produzidas com filme intermediário de $500 \mu \mathrm{m}$ de espessura. Ao contrário do que ocorre nas juntas com filme de $100 \mu \mathrm{m}$, o filme intermediário não é expulso do centro das juntas produzidas com filmes de $500 \mu \mathrm{m}$ de espessura. Na Figura 7A é possível identificar a presença de filme intermediário no centro das juntas (região I da Figura 5B). Neste caso, o calor fricional gerado não foi suficiente para diminuir significativamente a viscosidade do filme intermediário a fim de possibilitar sua completa expulsão para as extremidades da junta. Adicionalmente, observa-se que as irregularidades produzidas pelo pré-tratamento superficial na superfície do alumínio foram eficientemente preenchidas com o filme amolecido durante o processo de união. Assim, efetivo micro-ancoramento é estabelecido na interface alumínio-filme. Por outro lado, na interface filme-compósito identificou-se uma linha de separação constante que se estende desde regiões centrais (Figura 7B) até periféricas (Figura 7C) da junta. Considerando a baixa condutividade térmica do PPS $\left(0,19 \mathrm{~W} \mathrm{~m}^{-1} \mathrm{~K}^{-1}[25]\right)$, o calor friccional gerado na superfície do alumínio não foi suficiente para amolecer/fundir eficientemente o filme e a matriz do compósito. O amolecimento parcial do filme intermediário permite que este se conforme sobre as irregularidades da superfície pré-tratada do compósito devido à pressão exercida durante o processo de união (Figura 7C). No entanto, a interdifusão das cadeias de PPS foi aparentemente impossibilitada e, consequentemente, a união entre tais componentes após a consolidação da junta foi prejudicada.

(A)

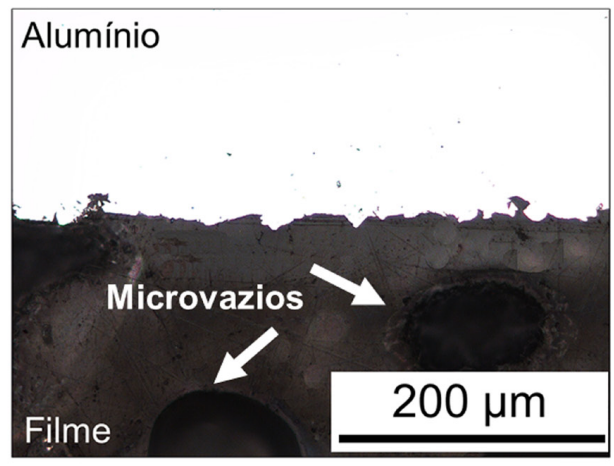

(B)

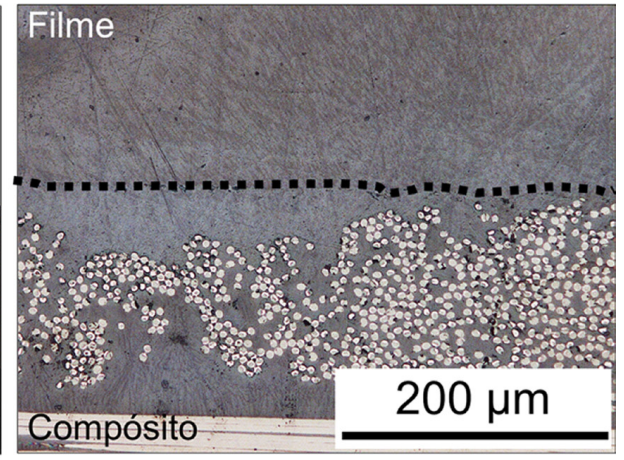

(C)

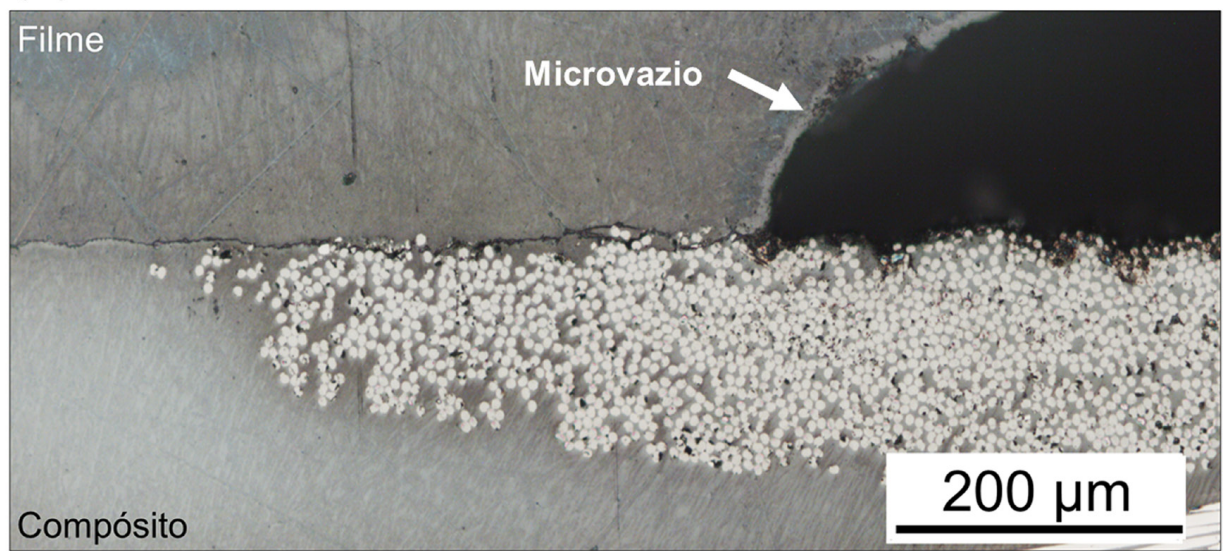

Figura 7. Detalhes microestruturais da interface de juntas unidas por FSpJ com filme intermediário de $500 \mu \mathrm{m}$ de espessura. (A) Região I, (B) região II e (C) região III da Figura 5B.

Adicionalmente, nota-se a presença de microvazios nos componentes poliméricos (compósito e filme) das juntas produzidas com ambas as espessuras de filme, conforme indicado nas Figuras 6 e 7. A formação de tais microvazios se dá durante o processo de união, quando o PPS amolecido pelo calor friccional é submetido ao fluxo pelo movimento axial da ferramenta. Assim, bolhas de ar são aprisionadas na massa polimérica dando origem aos 
microvazios depois da consolidação da junta. A formação de microvazios e outros defeitos volumétricos em juntas unidas por FSpJ é descrita em detalhes por Goushegir et al. [9] e André et al. [21]. Ressalta-se ainda que não foram observadas evidências de correlação entre a formação de microvazios e a ocorrência de processos degradativos induzidos pelo processo de união FSpJ, uma vez que investigações recentes descartaram a possibilidade de extensiva degradação térmica dos componentes poliméricos das juntas durante a união [30].

\subsection{Desempenho mecânico}

O desempenho mecânico das juntas unidas por FSpJ com diferentes espessuras de filme intermediário foram avaliadas através de ensaios de resistência ao cisalhamento sob tração. A Figura 8 apresenta exemplos de curvas força $\times$ deslocamento típicas das juntas híbridas unidas por FSpJ com diferentes espessuras de filme intermediário.

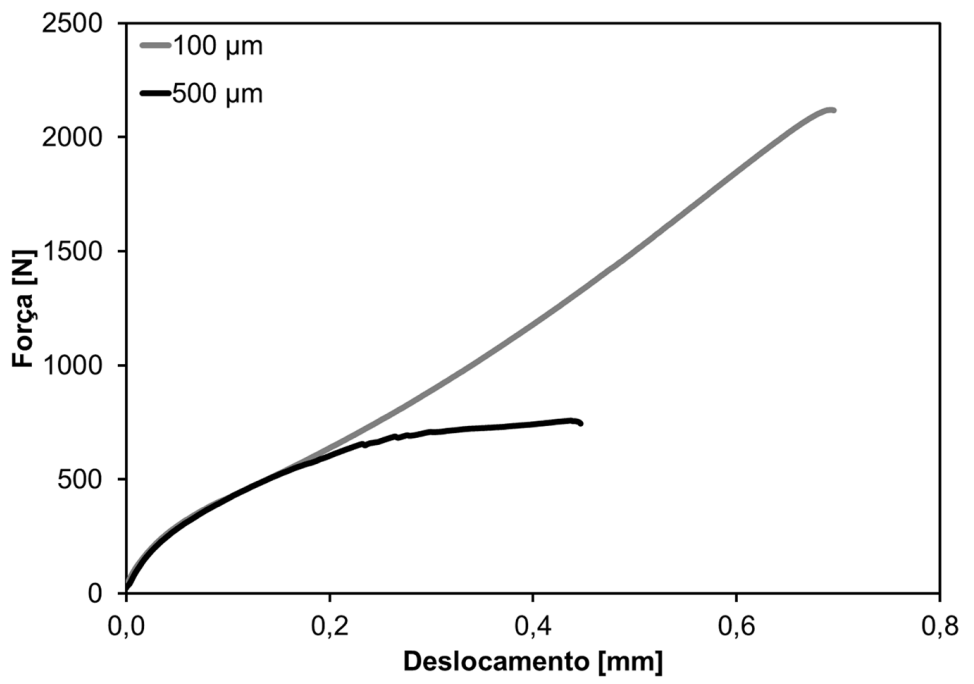

Figura 8. Exemplos de curvas força $\times$ deslocamento obtidas em ensaio de cisalhamento sob tração típicas das juntas híbridas unidas por FSpJ com filme intermediário com espessuras de 100 e $500 \mu \mathrm{m}$.

A Figura 9 compara os valores de força máxima de cisalhamento sob tração e deslocamento na ruptura de juntas unidas por FSpJ com diferentes espessuras de filmes intermediários e juntas sem filme. As juntas produzidas com filme de $100 \mu \mathrm{m}$ de espessura apresentaram maiores valores de força máxima de cisalhamento $(2093 \pm 180 \mathrm{~N})$ e maiores valores de deslocamento $(0,72 \pm 0,07 \mathrm{~mm})$. As juntas produzidas com filme mais espesso $(500 \mu \mathrm{m})$ apresentaram rigidez inicial bastante similar às juntas produzidas com o filme de $100 \mu \mathrm{m}$ de espessura. No entanto, comparativamente às juntas com filme mais fino, as juntas com filme mais espesso $(500 \mu \mathrm{m})$ apresentaram menores valores de deslocamento $(0,47 \pm 0,03 \mathrm{~mm})$ e menores valores de força máxima de cisalhamento $(708 \pm 69 \mathrm{~N})$.

As juntas produzidas com filme de $500 \mu \mathrm{m}$ apresentaram apenas $34 \%$ da resistência ao cisalhamento das juntas produzidas com filme de $100 \mu \mathrm{m}$. Como discutido na Seção 4.2, o filme mais fino (100 $\mu \mathrm{m})$ é amolecido com maior eficiência do que o filme mais espesso $(500 \mu \mathrm{m})$ pelo calor friccional gerado durante o processo de união. Dessa forma, apesar da interface alumínio-filme apresentar efetivo micro-ancoramento para ambas as juntas, a interface filme-compósito apresenta-se mais coesa para as juntas com filme de $100 \mu \mathrm{m}$. A melhor eficiência do amolecimento deste filme intermediário favorece a interdifusão das cadeias de PPS da matrix do compósito e do filme, como observado na Figura 6A.

Adicionalmente, a Figura 10 apresenta as superfícies de fratura de juntas unidas por FSpJ com filmes de 100 e $500 \mu \mathrm{m}$ de espessura. Goushegir et al. [9] propôs um modelo para elucidar os mecanismos de união e falha de juntas híbridas unidas por FSpJ. De forma simplificada, a área de união das juntas pode ser dividida em 


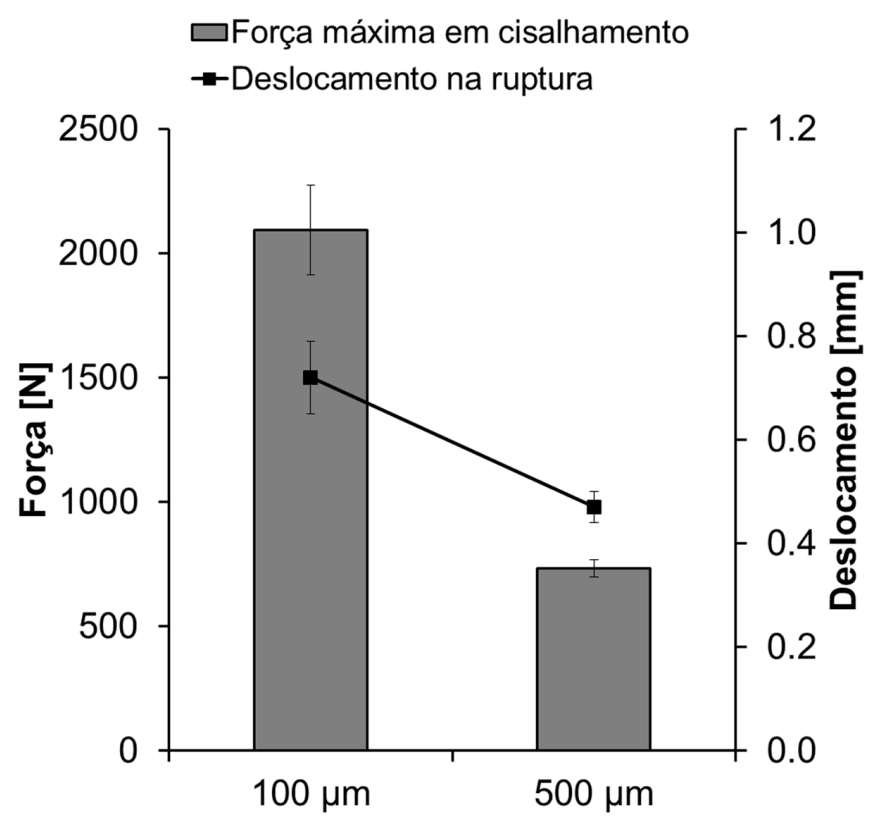

Figura 9. Comparação entre os valores de força máxima de cisalhamento e respectivos deslocamentos máximos na ruptura de juntas híbridas unidas por FSpJ com diferentes espessuras de filme intermediário.

(A)

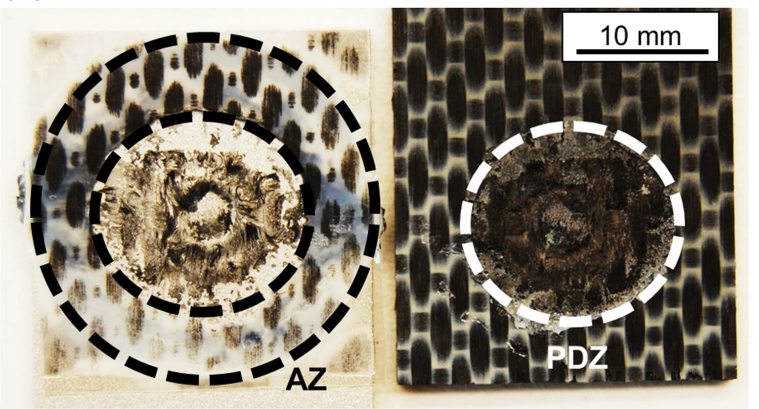

(B)

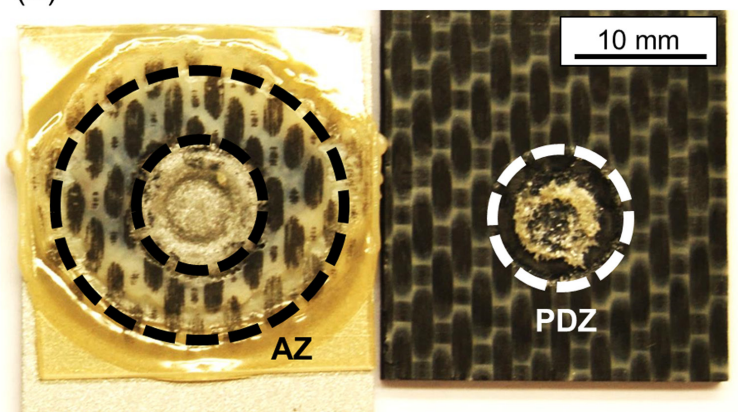

Figura 10. Superfícies de fratura de juntas unidas por FSpJ com filmes de (A) $100 \mu \mathrm{m}$ e (B) $500 \mu \mathrm{m}$ de espessura. As linhas pontilhadsa indicam a PDZ e a AZ das juntas.

três zonas: zona plasticamente deformada (PDZ), zona adesiva (AZ) e zona de transição (TZ). Em contraste ao modelo proposto para as juntas sem filme, a TZ não foi identificada nas juntas com filme intermediário. Tal zona caracteriza-se por um elevado volume de bolhas de ar aprisionadas entre PDZ e AZ. No entanto, com a adição do filme intermediário essas bolhas de ar não foram observadas. Assim, para as juntas com filme intermediário foram identificadas apenas duas zonas bem definidas: PDZ e AZ. Como proposto por Goushegir et al. [9], a PDZ corresponde à região central da junta e é a zona mais resistente das juntas unidas por FSpJ devido ao contato íntimo entre alumínio e compósito e à formação do cerne metálico nesta região. A AZ corresponde à região periférica da junta, onde o principal mecanismo de união são as forças adesivas fornecidas pelo filme intermediário reconsolidado. O filme de PPS é amolecido durante o FSpJ e preenche as irregularidades na superfície do alumínio e do compósito, estabelecendo micro-ancoramentos nas interfaces alumínio-filme e filme-compósito. As superfícies de fratura exibem o filme ancorado na superfície do alumínio depois do ensaio de cisalhamento. Além disso, observa-se forte interação entre filme e compósito na AZ através das impressões de fibra de carbono na superfície do filme (Figura 10). 
Influência da Espessura do Filme Polimérico Intermediário na Resistência Mecânica de Juntas Híbridas de Alumínio 2024-T3 e CF-PPS Produzidas por União Pontual por Fricção

As superfícies de fratura revelaram PDZ duas vezes maiores para as juntas com filmes de $100 \mu \mathrm{m}\left(16 \pm 2 \mathrm{~mm}^{2}\right)$ contra $7 \pm 1 \mathrm{~mm}^{2}$ para as juntas com filme de $500 \mu \mathrm{m}$ de espessura (Figura 10). Para o filme mais fino, a eficiência de amolecimento devido ao calor gerado por fricção durante o processo FSpJ é maior. Assim, a baixa viscosidade atingida com a fusão do filme favorece sua expulsão do centro para as extremidades da junta, promovendo um contato íntimo entre compósito e alumínio nesta região. Além disso, a interdifusão de cadeias de PPS entre filme e compósito é favorecida pela menor espessura do filme intermediário (Figura 6A). Adicionalmente, maiores AZ foram produzidas com filme de $100 \mu \mathrm{m}$ de espessura. Diante da baixa condutividade térmica do PPS, o filme de $500 \mu \mathrm{m}$ é apenas parcialmente amolecido durante o processo de união. Dessa forma, sua capacidade de molhar e preencher as irregularidades nas superfícies dos componentes para o estabelecimento de micro-ancoramento e forças adesivas é reduzida. Como resultado, tem-se zonas adesivas menores ( $\left.33 \pm 1 \mathrm{~mm}^{2}\right)$. Por outro lado, o filme de $100 \mu \mathrm{m}$ é extensivamente amolecido durante o processo de união, produzindo zonas adesivas de $38 \pm 1 \mathrm{~mm}^{2}$. Assim, a utilização do filme mais fino resulta em juntas com maiores áreas de união e, consequentemente, melhor adesão entre as partes. Ademais, a formação do cerne metálico é favorecida pelo uso do filme mais fino, como discutido na Seção 4.2 (Figura 5). Dessa forma, a combinação de tais mecanismos leva à resistência mecânica superior para as juntas híbridas produzidas com filme intermediário de $100 \mu \mathrm{m}$ de espessura.

Em comparação às juntas sem filme, as juntas produzidas neste trabalho com filme de $100 \mu \mathrm{m}$ de espessura apresentaram acréscimo de $18 \%$ na força máxima de cisalhamento (2093 \pm 180 contra $1774 \pm 192 \mathrm{~N}$ ) e de $7 \%$ no deslocamento na ruptura $(0,72 \pm 0,07$ contra $0,67 \pm 0,11 \mathrm{~mm})$. Todavia, o aprimoramento do pré-tratamento superficial do componente compósito pode incrementar significativamente o desempenho mecânico das juntas com filme intermediário frente às juntas sem filme. Investigações recentes demonstraram acréscimos de até $55 \%$ em força máxima de cisalhamento. Adicionalmente, o alongamento na ruptura alcançou incrementos de até $11 \%$ em comparação às juntas sem filme. Tal aprimoramento do desempenho mecânico das juntas deve-se à contribuição do filme intermediário no aumento da área de união das juntas unidas por FSpJ. No entanto, a análise completa dessa comparação entre juntas com e sem filme está fora do escopo deste trabalho e será discutida detalhadamente em publicações posteriores.

\section{Conclusões}

Este estudo avaliou a influência da espessura de filme de PPS intermediário no desempenho mecânico de juntas de AA2024-T3/CF-PPS. Duas espessuras de filme foram analisadas, $100 \mu \mathrm{m}$ e $500 \mu \mathrm{m}$. Dentre as principais conclusões, tem-se que:

- A espessura do filme influencia diretamente na formação do macro-ancoramento mecânico entre os componentes. A junta com filme mais fino $(100 \mu \mathrm{m})$ apresentou ancoramento mecânico eficiente. $\mathrm{O}$ cerne metálico dessa junta alcançou a matriz polimérica do compósito, enquanto que o cerne da junta com filme de $500 \mu \mathrm{m}$ de espessura inseriu-se apenas no filme intermediário.

- No caso do filme com 500 um de espessura, filme e compósito foram unidos apenas pela pressão exercida durante o processo de união. A interface filme-compósito apresentou uma linha de separação constante ao longo da seção transversal da junta indicando que o calor friccional gerado foi insuficiente para promover amolecimento considerável do filme e consequente interdifusão das cadeias de PPS entre o filme e o compósito. Tal linha foi identificada parcialmente nas juntas com filme de $100 \mu \mathrm{m}$. Em regiões centrais da junta a linha de separação não é percebida, sugerindo a ocorrência da interfidusão de cadeias poliméricas nesta região para as juntas com filme de $100 \mu \mathrm{m}$ de espessura.

- As juntas com filmes de $100 \mu \mathrm{m}$ apresentaram força máxima de cisalhamento (2093 $\pm 180 \mathrm{~N}$ ) cerca de três vezes maior que as juntas com filme de $500 \mu \mathrm{m}$ (708 $\pm 69 \mathrm{~N}$ ).

- As superfícies de fratura das juntas revelaram áreas de união maiores para as juntas com filmes de $100 \mu \mathrm{m}$ (53 \pm 2 contra $40 \pm 1 \mathrm{~mm}^{2}$ ).

Portanto, concluiu-se que dentre as espessuras de filme investigadas, a adição do filme mais fino $(100 \mu \mathrm{m})$ resultou em juntas mais resistentes em comparação à adição do filme de $500 \mu \mathrm{m}$. Novos estudos utilizando o Método dos Elementos Finitos (MEF) deverão complementar esta análise na busca pela espessura do filme intermediário que maximiza a resistência mecânica de juntas híbridas unidas por FSpJ. 


\section{Agradecimentos}

Os autores agradecem à Helmholtz Association da Alemanha pelo suporte financeiro (Young Investigator Group "Advanced Polymer-Metal Hybrid Structures" (Grant No. VH-NG-626), à FAPESP pela bolsa de mestrado concedida à Natália M. André (Processo 2014/09271-0) e ao CNPq pela bolsa de produtividade em pesquisa concedida ao Leonardo B. Canto (Processo 304169/2014-5).

\section{Referências}

[1] Amancio-Filho ST, Santos JF. Joining of polymers and polymermetal hybrid structures: recent developments and trends. Polymer Engineering and Science. 2009;49(8):1461-1476. http:// dx.doi.org/10.1002/pen.21424

[2] Mallik PK. Materials, design and manufacturing for lightweight vehicles. 1st ed. Cambridge: Woodhead Publishing Limited; 2010. http://dx.doi.org/10.1533/9781845697822.

[3] Boeing. Boeing 787: from the ground up. Aero. 2006;(24):132. [access june 2015]. Available from: www.boeing.com/ commercial/aeromagazine/articles/qtr_4_06/article_04_2. html

[4] Flight Airworthiness Support Technology-FAST. Airbus Technical Magazine. 2011;48:1-40.

[5] Composites World. TenCate Advanced Armour will provide ballistic solutions for the Embraer KC-390. Cincinnati; 2013. [access june 2015]. Available from: http://www.compositesworld. com/news/tencate-advanced-armour-will-provide-ballisticsolutions-for-the-embraer-kc-390

[6] SAE International. BMW i3, the inside story: what it's made of how it's made. USA; 2013. [access june 2015]. Available from: http://articles.sae.org/12056/

[7] Abibe AB, Santos JF, Amancio-Filho ST. Friction staking: a nova staking joining method for hybrid structures. In: Proceedings of the Annual Technical Conference of Society of Plastics Engineers (ANTEC 2014); 2014; Las Vegas, Nevada. USA: Society of Plastics Engineers; 2014.

[8] Blaga L, Bancilă R, Santos JF, Amancio-Filho ST. Friction Riveting of glass-fibre-reinforced polyetherimide composite and titanium grade 2 hybrid joints. Materials \& Design. 2013;50:825-829. http://dx.doi.org/10.1016/j.matdes.2013.03.061.

[9] Goushegir SM, Santos JF, Amancio-Filho ST. Friction spot joining of Aluminum AA2024/carbon-fiber reinforced poly(phenylene sulfide) composite single lap joints: microstructure and mechanical performance. Materials \& Design. 2014;50:196-206. http:// dx.doi.org/10.1016/j.matdes.2013.08.034

[10] Marinelli JM, Lambing CLT. Advancement in welding technology for composite-to-metallic joints. Journal of Advanced Materials. $1994 ; 25: 20-27$

[11] Balle F, Emrich S, Wagner G, Eifler D, Brodyanski A, Kopnarski M. Improvement of ultrasonically welded aluminum/carbon fiber reinforced polymer-joints by surface technology and high resolution analysis: improvement of al/cfrp-joints by surface technology. Advanced Engineering Materials. 2013;15(9):814820. http://dx.doi.org/10.1002/adem.201200282.

[12] Messler RW, Bell J, Craigue O. Laser beam weld bonding of AA5754 for automobile structures. Welding Journal. 2003;82:151s-159s.

[13] Mitschang P, Velthuis R, Didi M. Induction spot welding of metal/CFRPC hybrid joints. Advanced Engineering Materials. 2013;15(9):804-813. http://dx.doi.org/10.1002/adem.201200273.

[14] Rotheiser J. Joining of plastics. 2nd ed. Munich: Hanser Publisher; 2004.

[15] Chang B, Shi Y, Dong S. Comparative studies on stresses in weldbonded, spot-welded and adhesive-bonded joints. Journal of
Materials Processing Technology. 1999;87(1-3):230-236. http:// dx.doi.org/10.1016/S0924-0136(98)00355-0.

[16] Campilho RDSG, Pinto AMG, Banea MD, da Silva LFM. Optimization study of hybrid spot-welded/bonded single-lap joints. International Journal of Adhesion and Adhesives. 2012;37:86-95. http:// dx.doi.org/10.1016/j.ijadhadh.2012.01.018.

[17] Xu W, Chen DL, Liu L, Mori H, Zhou Y. Microstructure and mechanical properties of weld-bonded and resistance spot welded magnesium-to-steel dissimilar joints. Materials Science and Engineering. 2012;537:11-24. http://dx.doi.org/10.1016/j. msea.2011.12.096

[18] Amancio-Filho ST, Santos JF. Verfahren und Vorrichtung zum Verbinden von Kunststoff- und Metallteilen. EP 2329905 B1. 2012 May 30.

[19] Amancio-Filho ST, Bueno C, Santos JF, Huber N, Hage E Jr. On the feasibility of friction spot joining in magnesium/fiber-reinforced polymer composite hybrid structures. Materials Science and Engineering A. 2011;528(10-11):3841-3848. http://dx.doi. org/10.1016/j.msea.2011.01.085.

[20] Esteves JV, Goushegir SM, Santos JF, Canto LB, Hage E Jr, Amancio-Filho ST. Friction spot joining of aluminum AA6181-T4 and carbon fiber-reinforced poly(phenylene sulfide): effects of process parameters on the microstructure and mechanical strength. Materials \& Design. 2015;66:437-445. http://dx.doi. org/10.1016/j.matdes.2014.06.070.

[21] André NM, Goushegir SM, Canto LB, Santos J, Amancio ST. On the microstructure and mechanical performance of Friction Spot Joining with additional film interlayer. In: Proceedings of the Annual Technical Conference of Society of Plastics Engineers (ANTEC 2014); 2014; Las Vegas, Nevada. USA: Society of Plastics Engineers; 2014. p. 1791-1797.

[22] Esteves JV, Amancio-Filho S, Santos J, Canto L, Hage E Jr. Friction Spot Joining of aluminum 6181-T4 and carbon fiber reinforced poly(phenylene sulfide). In: Proceedings of the Annual Technical Conference of Society of Plastics Engineers (ANTEC 2012); 2012; Orlando, USA. 2012. USA: Society of Plastics Engineers; 2012.

[23] Davis JR. Aluminum and aluminum alloys. Materials Park: ASM International; 1993.

[24] Tencate Advanced Composites. CETEX ${ }^{\circledR}$ PPS. Holanda; 2009. Technical datasheet.

[25] Lite P. PPS film. Austria; 2009. Technical datasheet.

[26] Amancio-Filho ST, Camillo APC, Bergmann L, Santos JF, Kury SE, Machado NGA. Preliminary investigation of the microstructure and mechanical behaviour of 2024 aluminium alloy friction spot welds. Materials Transactions. 2011;52(5):985-991. http:// dx.doi.org/10.2320/matertrans.L-MZ201126.

[27] Budgell DR, Day M, Cooney JD. Thermal degradation of poly(phenylene sulfide) as monitored by pyrolysis- GC/MS. Polymer Degradation \& Stability. 1994;43(1):109-115. http:// dx.doi.org/10.1016/0141-3910(94)90232-1.

[28] Jachowicz J, Kryszewski M, Sobol A. Thermal degradation of poly(2-methylphenylene oxide), poly(2,5-dimethylphenylene 
Influência da Espessura do Filme Polimérico Intermediário na Resistência Mecânica de Juntas Híbridas de Alumínio 2024-T3 e CF-PPS Produzidas por União Pontual por Fricção

oxide) and poly(1,4-phenylene oxide). Polymer. 1979;20(8):9951002. http://dx.doi.org/10.1016/0032-3861(79)90198-8.

[29] Sinmazçelik T. Natural weathering effects on the mechanical and surface properties of polyphenylene sulphide (PPS) composites. Materials \& Design. 2006;27(4):270-277. http:// dx.doi.org/10.1016/j.matdes.2004.10.022.
[30] André NM, Goushegir SM, Santos J, Amancio-Filho ST, Canto LB. Influência termomecânica do processo de União Pontual por Fricção na recristalização dos componentes poliméricos de junta híbrida CF-PPS/Alumínio com filme intermediário de PPS. In: Anais do $13^{\circ}$ Congresso Brasileiro de Polímeros; 2015; Natal, RN. São Carlos: ABPol; 2015. 